\title{
ESTUDOS COMPARADOS, PROJETO HISTÓRICO E ANÁLISE DE POLÍTICAS PÚBLICAS: ENTREVISTA COM HUGO ZEMELMAN*
}

\author{
Nora Rut KraWCZYK ${ }^{* *}$ \\ Reginaldo C. CorrêA DE Moraes ${ }^{* * *}$
}

\begin{abstract}
RESUMO: Conhecido pelo seu vasto conhecimento e experiência nas áreas da epistemologia e das políticas sociais, o professor Hugo Zemelmam aborda nesta entrevista os problemas metodológicos envolvidos nas análises políticas que trabalham com a noção de comparação nos processos históricos. Salienta o desafio da construção de um conhecimento sobre a realidade latino-americana que incorpore a historicidade dos diferentes processos nacionais, isto é, a complexidade dos fenômenos políticos, sociais, culturais e/ou econômicos no momento em que eles são abordados, e que não se restrinja à comparação de indicadores ou à elaboração de analogias, tentação, a seu ver, enganadora, na qual teriam caído muitos estudos comparados produzidos nos últimos anos.
\end{abstract}

Palavras-chave: Políticas sociais. Processo de conhecimento. Estudos comparados. América Latina.

\section{Compared STUdies,}

HISTORICAL PROJECT AND A PUBLIC POLICIES ANALYSIS:

AN INTERVIEW WITH HUGO ZEMELMAN

ABSTRACT: Renowned for his huge knowledge and experience in the fields of epistemology and social policies, professor Hugo Zemelmam tackles the methodological problems involved in the political analyses that compare historical processes. As for the Latin American reality, he

* As inicias (E) e (HZ) no início dos parágrafos indicam, respectivamente, as perguntas dos entrevistadores e as respostas de Hugo Zemelman.

** Professora doutora no Programa de Estudos Pós-graduados em Educação: Currículo, da Universidade Católica de São Paulo (PUC-SP) e na Faculdade de Educação da Universidade Estadual de Campinas (UNICAMP).E-mail: norak@terra.com.br

*** Professor doutor no Departamento de Ciência Política da Universidade Estadual de Campinas (UNICAMP).E-mail: remoraes@terra.com.br 
highlights how challenging it is to build knowledge that takes in consideration the historicity of the different national processes, that is, the complexity of the political, social, cultural and/or economical phenomena of the moment they are dealt with. Such knowledge should not limit to comparing indicators or elaborating analogies, a temptation he considers as deceiving and that spoils many compared studies produced lately.

Key words: Social policies. Knowledge process. Compared studies. Latin America.

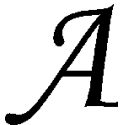

entrevista que transcrevemos a seguir foi concedida pelo sociólogo chileno, radicado no México, Hugo Zemelman, por ocasião de sua passagem por São Paulo, em setembro de 2002, onde participou no "Seminário Interdisciplinar Estado e Políticas Públicas na América Latina" (PUC-SP). Esse encontro teve a finalidade de analisar as perspectivas da América Latina nos campos sócio-político-cultural, as convergências e divergências que identificam cada Estado-Nação e as dificuldades de realizar estudos comparativos. Os convidados estrangeiros para o encontro foram Zemelman e o cientista político argentino Atílio Borón.

O professor Zemelman é, há vários anos, professor e pesquisador do El Colégio de México e coordenador do Grupo de Teoria e Método do Conselho Latino-americano de Ciências Sociais (CLACSO). É também Assessor Geral da Pós-graduação em Humanidades e Ciências Sociais da Universidad de la Ciudad de México. Publicou numerosos livros e artigos sobre teoria política e epistemologia das ciências sociais, tais como: Historia y Política en el conocimiento (1979); Estado, poder y lucha política (1986); De la história a la Política - La experiencia de América Latina (1989); Los horizontes de la razón (1992); Subjetividad: umbrales del pensamiento social ( 1997); Necesidad de conciencia (2002).

Conhecido pelo seu vasto conhecimento e experiência nas áreas da epistemologia e das políticas sociais, Zemelmam abordou, no encontro e na entrevista adiante reproduzida, os problemas metodológicos envolvidos nas análises políticas que trabalham com a noção de comparação nos processos históricos.

A trajetória e as obras de Zemelman deixam entrever a sua preocupação permanente por compreender o conhecimento nas suas implicações ético-políticas e como processo de relação com o mundo.

Nesta entrevista, fica evidente a sua preocupação em propor o desafio da construção de um conhecimento sobre a realidade latinoamericana que incorpore a historicidade dos diferentes processos nacio- 
nais, isto é, a complexidade dos fenômenos políticos, sociais, culturais el ou econômicos no momento em que eles são abordados, e não se restringia à comparação de indicadores ou à elaboração de analogias, tentação, a seu ver, enganadora, na qual teriam caído muitos estudos comparados produzidos nos últimos anos.

$E:$ ¿El estudio comparado puede ser considerado más adecuado para el análisis de proyectos políticos?

$H Z$ : Lo primero que habría que señalar en relación con los estudios comparados son las diferentes funciones que esto puede cumplir. En este sentido podemos diferenciar entre estudios comparativos orientados a justificar opciones ideológicas, o bien estudios comparados orientados para determinar viabilidades concretas que se pueden contener de manera latente en una situación histórica.

La distinción anterior supone aclarar conceptualmente que los procesos asociados a políticas concretas deben ser analizados en sus determinaciones y sobredeterminaciones; esto es en su articulación dinámica y no como simples situaciones producidas por una lógica de factores encadenados, ninguno de los cuales aparece afectado por exigencias de especificidades históricas. De ahí que el desafío de la comparación la entendemos que reside en la capacidad de comparar la articulación que hace a un fenómeno, en cuanto a aquella que configura la especificidad de este. En efecto, lo que se compara es una situación de historicidad: esto es, como producto de procesos que han convergido en ella; pero también la historicidad entendida como latencia de posibilidades.

Otra línea de razonamiento a partir de lo anterior, refiere a lo que entendemos como modos de concreción de lo necesario en tanto posibilidad; a su vez, la posibilidad como concreción, pero la concreción como reflejo de inclusiones de niveles que cumplen la función de especificar la necesidad. Estas inclusiones las denominamos especificadoras y representan un modo de recuperar lo general como contorno del fenómeno que se muestra en contenidos particulares, pero que a la vez los rebasan como posibilidad de concreción. En este sentido, la concreción como incorporación del contorno puede interpretarse de una manera más abstracta en el siguiente enunciado: Lo que es como lo que está haciendo, y lo que está haciendo como articulación en su movimiento de cierres y aperturas al que está sometido el fenómeno particular.

En otras palabras, lo anterior podríamos esquematizarlo en la siguiente secuencia epistémico-metodológica que requiere de desarrollos puntuales: 
i) Los modos de concreción como inclusiones ya incluidas en un momento, lo que supone tener claro a este como universo de significaciones y, en consecuencia, de relaciones;

ii) La inclusión anterior permanece siempre abierta a posibles nuevas inclusiones en función de las necesidades de relaciones del fenómeno. Es lo que denominamos inclusividad; lo que supone saber leer al momento como universo de significaciones que están implicando relaciones necesarias entre varios planos de la realidad, a partir del propio problema que interesa en tanto eje de razonamiento;

iii) La inclusividad anterior en base a lo necesario se corresponde a la idea de lo general pero que se muestra en contenidos particulares;

iv) Los contenidos propios de la inclusividad están siempre, por exigencia lógico-epistémica, rebasados por un contorno que es la inclusividad hecha posible a partir de determinadas prácticas, o bien de planteamientos teóricos o ideológicos; lo que de alguna manera se puede relacionar con la problemática vinculada con las lecturas alternativas de una situación dada;

v) Por último lo que es como lo que está siendo en tanto totalización abierta.

Lo que está implícito en lo anterior es la necesidad, desde la exigencia de la historicidad, de proceder de una descomposición de los córpora teórico. En este sentido, me permito leer a usted algunas reflexiones escritas a este respecto:

El distanciamiento respecto de los encuadres teóricos del razonar se cimenta en la lógica de pensar desde lo externo y lo ignorado en base a relaciones posibles (todo fenómeno podrá ser definido como nivel dinámico de una articulación posible, cuya función será problematizar cualquier organización teórica "cerrada" que desconozca las exigencias lógico epistemológicas).

El uso de los conceptos exige ser historizado, en el sentido de que deben ser definidos en función de la articulación y no de la función de explicación implícita en la teoría de origen. Los conceptos son objeto de una problematización para determinar su especificidad en función de la articulación. La explicación es absorbida al interior de un campo de observación que define las bases para una nueva teorización. 
No se trata de "probar" un concepto sino de descubrir su especificidad al interior de la articulación. La diferencia consiste en que los conceptos no buscan garantías de que su contenido se contraste con determinadas estructuras reales; sino de que el contenido sea determinable según la exigencia de especificidad creciente, que transformará el contenido definido por la pertenencia a un hábeas teórico. De manera que si adoptamos el planteamiento de la contrastación con lo real-objetivo, ésta tendrá que interpretarse como la adecuación a la exigencia de especificidad creciente, según es definida por la función lógico epistemológica de la articulación. (Confrontar uso crítico de la teoría: Apartado: pasos para la descomposición de los hábeas teóricos, El Colegio de México, p. 149-150)

E: ¿En América Latina la investigación educacional crítica fue cada vez más alejándose del enfoque comparativo debido a su fuerte asociación con el paradigma posibilista y con la política imperialista de desarrollo. Los estudios comparados pueden adquirir otro sentido epistemológico que escape de ese destino. Es posible pensar en una perspectiva histórico-sociológica?

$H Z$ : Pensamos que supone un razonamiento de articulación al interior de campos de significación histórico-sociales; lo que supone como desafío que enfrentado al problema del recorte este debe ser de una articulación en tanto concreción especificadora, esto es que a pesar de su delimitación el recorte debe incluir distintos niveles tanto aquellos que están más directamente vinculados con el problema como los que están en una relación mediata, pudiendo considerarse que están fuera de sus limites. Es precisamente en relación con un problema de esta naturaleza donde se plantea el problema anterior de las inclusiones.

En el marco de esta pregunta cabe incorporar la idea, ya mencionada, de los campos de significación histórico-sociales. La función de estos es planearnos exigencias de articulación desde lo necesario, según resulte de una problematización del momento histórico del que situamos el problema, aunque estas no se materialicen concretamente. Lo que no podemos olvidar es la relación entre niveles y momentos fundamental si concebimos al problema, más allá de su condición morfológica de producto, como un proceso.

E: ¿Estudios comparados dirigidos a entender o a evaluar políticas públicas: suponen ellos unidades que mantienen entre si relaciones de semejanza o de pertinencia en un mismo campo (niveles de desarrollo, padrones sociales, tradiciones culturales)?

$H Z$ : Respecto de las unidades de análisis, nos planteamos el problema de la homogenización de la realidad cuando la leemos en función 
de ciertos límites. Esta aseveración debe ser corregida siempre que observemos al problema desde sus exigencias dinámicas, esto es, desde el ángulo de procesos que constituyen el producto, trascendiendo su condición de producto medido o medible.

Lo anterior exige comprender las unidades de análisis como recortes que, organizados en función de determinados límites empíricos o conceptuales, debe incorporar los procesos constitutivos; de ahí que sus elementos componentes deben dar cuenta del movimiento del límite: de lo dándose en lo dado. Esto es, construir la articulación en función de una doble exigencia: la articulación en función de lo articulado históricamente, en una situación dada; y la articulación en función de lo articulable según las exigencias del momento entendido en términos secuénciales, esto es, en la perspectiva del tiempo longitudinal. En pocas palabras, lo anterior se podría condensar en el concepto de historicidad.

En efecto, "una de las formas para organizar un acercamiento hacia la realidad puede ser el razonamiento por inclusiones crecientes y necesarias de elementos. Sin embargo, esta inclusión no siempre sirve de premisa para derivar conclusiones cualitativas. Esto ocurre cuando la inclusión consiste en simple agregación, pues en ese caso no permite otro tipo de conclusiones que la contenida en la propia descripción morfológica de los datos (...)".

"De esto se desprende que es imposible resolver el problema de la derivación cualitativa desde datos estadísticos agregados, a menos que se consideren las mediaciones que operan entre la premisa numérica y los efectos cualitativos; mediaciones que se refieren a la constitución de la realidad, lo que consideramos como una condición previa a la operacionalización" (Mayores desarrollos sobre el tema los he incorporado en un artículo titulado: La relación de conocimiento y el problema de la objetividad de los datos. Estudios Sociológicos, vol. 11, n. 33, Sept.-Dic 1993)

$E$ : ¿Cuáles son los problemas de método que surgen, en los estudios comparados, cuando pensamos en la construcción de indicadores?

$H Z$ : En relación al problema de los indicadores creemos que se debe dar una discusión orientada a clasificarlos en función de criterios dinámicos. A manera de ejemplo podríamos señalar criterios como los siguientes:
a) Indicadores de proceso;
b) Indicadores de resultado; 
c) Indicadores de relaciones;

d) Indicadores de puntos de articulación entre niveles de la realidad.

Esta clasificación podría operar tanto a niveles micro como macrosociales, como también ajustarse para manejarse para dar cuenta de situaciones estructurales como coyunturales.

El problema de fondo en la construcción de indicadores es poder determinar con ellos el campo de opciones viables, de manera de poder delimitar las potencialidades y direccionalidades posibles que se contienen en la articulación con mayor probabilidad de transformarse en proyecto de desarrollo. Lo medular es identificar la relación que las diferentes proposiciones estratégicas mantienen entre sí, tanto lo relativo al ordenamiento interno de los indicadores, como especialmente a la articulación de la base de realidad que se toma como referente para las decisiones. La reconstrucción de las estrategias, a partir de sus indicadores, debe reflejar la relación de posibilidad objetivo de cada alternativa, en función de la presencia o ausencia de voluntades colectivas que representen una capacidad efectiva de intervención.

Lo que en resumen procuramos plantearnos es una suerte hermeneútica de los datos secundarios existentes. Propuesta que, como posibilidad, se asienta en la observación de que existen diferentes modalidades de construcción de los indicadores que dan cuenta de un producto o resultado, esto es, de los indicadores que constituyen los sistemas usuales de información. Éstas modalidades están asociadas con la flexibilidad que ofrece el dato construido en términos de posibilitar la construcción de indicadores articulados (ésta temática la he podido desarrollar con algún detalle en el trabajo titulado Crítica Epistemológica de los Indicadores. Jornada 114, El Colegio de México.)

E: ¿Cómo se colocan los estudios comparados - y qué problemas se presentan - cuando hay entre sus unidades de análisis relaciones de jerarquía?

$H Z$ : Respecto del problema de la relación entre unidades de análisis, considero necesario remplazar la idea de jerarquía con la de articulación especificadora. Pues no se trata simplemente de partir de relaciones asimétricas, sino más bien de situaciones historizadas, en forma de que las relaciones de simetría (de subalternidad, de explotación etc.) puedan analizarse en el conjunto de sus dimensiones según sean los campos de significación histórica que están comprometidos en esas relaciones. 
Por esta razón considero que la relación entre las unidades da análisis debe ser definida desde la perspectiva propia de las dinámicas articuladoras entre los componentes involucrados en cada una de ellas, incorporando sus implicaciones en lo que respecta a su dirección y tiempos.

$E$ : En los últimos años, los organismos internacionales reavivaron los estudios comparados, siguiendo la lógica funcionalista de los estudios de los años 50, para apontar déficits y la existencia de una homogeneidad de los problemas educacionales en la región. De ese modo, parecen unificar un cuadro de referencia conceptual acerca de las diferentes dimensiones de la problemática educativa y de sus prioridades e estratégias nacionales. Y parecen proponer reformas educativas también homogeneas y homogeneizantes. Los estudios criticos identifican la necesidad de investigaciones que permitan compreender la complejidad de los impactos produzidos por la política educativa implementada en América Latina, visualizando la interpenetración que ocurre entre los niveles globales y locales de la organización de las nuevas realidades educativas en los países latinoamericanos. Que perspectva teórico-metodológica e que catagorias de análisis permitirian estudiar los procesos de mediación en la concretización de los lineamientos de la reforma educativa y de las acciones impuestas en la región?

$H Z$ : Considero que tiene relación con una pregunta anterior, acerca de las "unidades que mantienen entre si relaciones de semejanza o de pertinencia en un mismo campo (niveles de desarrollo, padrones sociales, tradiciones culturales)": consideramos la necesidad de tomar en cuenta la articulación especificadora que se da en la relación entre niveles, pero pensada desde los dinamismos constitutivos de estos niveles. Metodológicamente supone orientarse en la perspectiva de rescatar las potencialidades ocultas de la realidad, recuperación de la historicidad sobre la tendencia a la generalización teórica. En este sentido, lo fundamental es captar las articulaciones ya que estas apuntan a nudos cuyo reconocimiento puede permitir definir espacios de potenciación por parte de los sujetos. De otro modo nos quedamos prisioneros de descripciones rigurosas, detallistas, pero estáticas. El problema de fondo podría también enunciarse de la siguiente manera: el conocimiento ya sea de cualquier problema social debe ir más allá de la simple descripción de objetos, de manera de poder profundizar en los espacios de posibilidades que se presentan o se ocultan en torno de ellos; espacios de posibilidades para los diferentes tipos de sujetos. 
E: Analizando los efectos del proceso de avance extraordinário de la globalización, en todas las dimensiones de la sociedad contemporánea, se puede constatar la tendencia contradictória de homogeneización de los procesos sociales y políticos y de diferenciación y profundización de los regionalismos y localismo. En los últimos años, muchos estudios han enfatizado la homogeneización de los procesos sociales, políticos y económicos y otros tienen elegido el camino de la revalorización de los particularismos. En que medida los estudios comparados podrian superar esta dicotomia?

$H Z$ : Me limito a decir que no creo que contribuya a resolver la dicotomía.

$E:$ ¿Un estudio comparado permitiría enriquecer las categorías de análisis para la mejor comprensión de cada una de las realidades nacionales?

HZ: La confrontación de especificidades, esto es, de situaciones historizadas como lo hemos planteado, puede permitir construir categorías pertinentes a cada contexto. Los procesos históricos hay que analizarlos en sus modos de concreción, atendiendo a cómo son afectados por las múltiples articulaciones que conforman el perfil de un momento histórico, no limitándose a describirlos a lo que tienen de general. En términos más amplios, lo que decimos plantea la problemática entre historicidad y legalidad. La historicidad apuntaría a lo que hemos llamado concreción de los procesos, según coordenadas de tiempo y espacio, aunque siempre analizadas en su secuencia longitudinal. Mientras que la legalidad podría estar apuntando a esta secuencia longitudinal elevada a rango, a veces ontológico, sin cuidar muchas veces de las exigencias epistémicas de la concreción en tiempo y espacio.

Cada situación histórica desde su especificidad se traduce en conceptos propios, lo que obliga a tener clara la siguiente distinción:

a) entre categorías de razonamiento que, en este caso, cumplen la función de vigilia para que se respeten determinadas exigencias dinámicas de la realidad socio-histórica, en cuanto a la necesidad de que tengan presencia de la construcción de problemas;

b) los conceptos en que se puede cristalizar el razonamiento epistémico cuando se pasa a la etapa de teorización.

Las consideraciones esquemáticas anteriores se formulan desde la exigencia de un esquema general metodológico en el que estamos 
trabajando, a partir de las premisas epistemológicas que hemos venido desarrollando en El Colegio de México. En términos sintéticos podríamos resumir este esquema en las siguientes proposiciones:

a) Todo producto o problema social que aparezca como estructurado debe ser leído desde sus dinámicas constitutivas;

b) Estas dinámicas constitutivas, o procesos, tienen lugar o se despliegan en diferentes niveles de la realidad, articulados entre sí; o bien, puede ocurrir que algunos de estos procesos se desplieguen involucrando a varios de estos niveles;

c) Las relaciones entre estos niveles, y su efecto en los procesos, determinan la especificidad de este;

d) Las relaciones aludidas no son mecánicas, sino que se caracterizan por conformar campos de relaciones internas que denominamos mediaciones de las relaciones entre niveles;

e) A partir de estas articulaciones de relaciones se pueden reconocer nudos de articulación, cuya importancia es que conforman los espacios desde los cuales se pueden potenciar los procesos en una u otra dirección.

Las consideraciones anteriores son un muy apretado resumen de problemas que exigen un mayor desarrollo. Considerando las limitaciones de esta entrevista me restrinjo a esbozar algunos lineamientos que pueden servir de base para argumentaciones que incorporen las fundamentaciones epistémicas fundamentales. No obstante, espero haber contribuido aunque someramente a un intercambio de ideas que, en el momento actual del desarrollo de las ciencias sociales, puede ser significativo para promover un debate orientado a enriquecer las formas de construcción de conocimiento socio-histórico.

Recebido e aprovado em fevereiro de 2003. 